\title{
Mediación pedagógica y conocimientos científicos que utilizan una muestra de docentes de ciencias en noveno año de dos circuitos del sistema educativo costarricense, para el desarrollo de competencias científicas
}

\author{
Pedagogical Mediation and Scientific Knowledge Using a Sample of Ninth Grade Science \\ Teachers in Two School Districts of the Costa Rica Educational System for the Development \\ of Scientific Competencies
}

\section{Mediação pedagógica e conhecimento científico utilizado por uma amostra de professores de ciências no nono ano de dois circuitos do sistema educacional da Costa Rica, para o desenvolvimento de competências científicas}

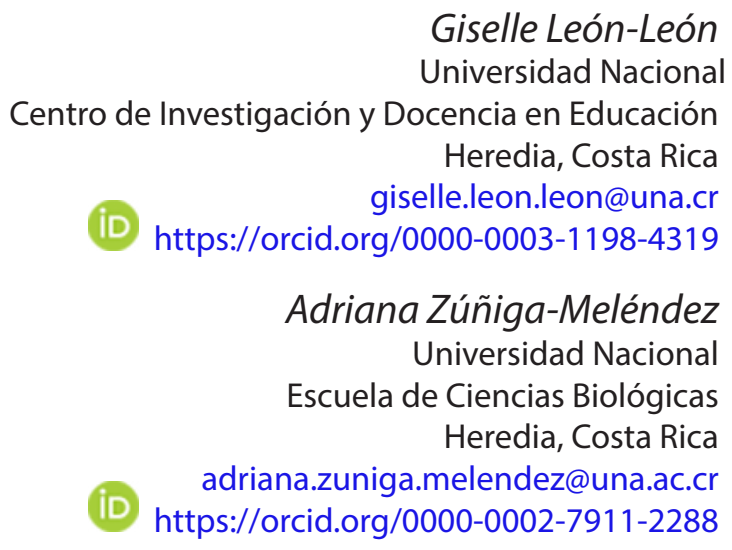

Recibido • Received • Recebido: 29 / 08 / 2017

Corregido • Revised • Revisado: 19/11/ 2018

Aceptado • Accepted • Aprovado: 05/ 04 / 2019

\begin{abstract}
Resumen: El artículo muestra un estudio realizado en el marco del proyecto interunidad de la Universidad Nacional, Costa Rica (UNA), titulado: “Las competencias científicas en estudiantes del tercer ciclo $\left(9^{\circ}\right)$ de educación general básica en la Región Central de Heredia circuito 01 y 02 de la Universidad Nacional" (Zúñiga y León 2017), sobre competencias científicas y mediación pedagógica. Específicamente este escrito se abocó al desarrollo del objetivo caracterizar la mediación pedagógica que utilizan docentes en su práctica cotidiana, para el desarrollo de las competencias científicas en estudiantes de $9^{\circ}$ año (de tercer ciclo de educación general básica). La investigación se fundamentó en el paradigma naturalista y una metodología que asume un tipo de investigación mixta, donde se contrastan datos cualitativos y cuantitativos a partir de la utilización de la observación y el cuestionario, para lograr un análisis más profundo. La investigación se efectuó con la colaboración de 17 docentes
\end{abstract}


doi: http://dx.doi.org/10.15359/ree.23-2.5

URL: http://www.una.ac.cr/educare

CORREO: educare@una.cr

de ciencias de la Regional de Heredia (división de zonas escolares, que propone el Ministerio de Educación de Costa Rica), anuentes a colaborar, por lo que los resultados obtenidos solo aplican a ese grupo, no se pueden generalizar. Dentro de los principales resultados se resalta que el rol, metodología (estrategias-recursos), acto educativo y evaluación utilizada por el colectivo docente del III ciclo tiende a estar más cercano a un modelo tradicional de enseñanza centrado en la transmisión de conocimientos. De igual forma, se evidencia que dado que la mediación principalmente se centra en el modelo tradicional, se priorizan, en la planificación, evaluación y mediación, conocimientos científicos conceptuales por encima de los procedimentales y actitudinales.

Palabras claves: Mediación pedagógica; conocimientos científicos; ciencias; secundaria; noveno; docentes; competencias científicas.

Abstract: This article presents the results of a study conducted within the framework of an interunit project of the National University of Costa Rica (UNA), titled "Las competencias científicas en estudiantes del tercer ciclo $\left(9^{\circ}\right)$ de educación general básica en la Región Central de Heredia circuito 01 y 02" (Zúñiga y León 2017), on scientific competencies and pedagogical mediation. Specifically, this paper focused on the objective of characterizing the pedagogical mediation that teachers use in their daily practice, for the development of scientific competencies in ninth grade students (corresponding to the third cycle of Costa Rican general basic education). The research was based on the naturalist paradigm and a methodology that assumes a type of mixed research design, where qualitative and quantitative data are compared with the use of observation and a questionnaire to achieve a deeper analysis. The research was conducted with the collaboration of 17 science teachers from Heredia School District (the administrative division of school zones proposed by the Ministry of Education of Costa Rica), willing to collaborate; so, to avoid generalizations, the results obtained only apply to that group. Among the main results, it is highlighted that the role, methodology (strategies and resources), educating act, and evaluation used by the teaching group of the third cycle tends to be closer to a traditional teaching model focused on the transmission of knowledge. Likewise, it is evident that, since mediation mainly focuses on the traditional model, conceptual and scientific knowledge is prioritized over procedural and attitudinal one in planning, evaluation, and mediation.

Keywords: pedagogical mediation; scientific knowledge; science; secondary school; ninth grade, teachers, scientific competences.

Resumo: $O$ artigo mostra um estudo realizado no âmbito de um projeto inter-unidades da Universidade Nacional da Costa Rica (UNA) titulado: "Las competencias científicas en estudiantes del tercer ciclo ( $\left.9^{\circ}\right)$ de educación general básica en la Región Central de Heredia circuito 01 y 02" (Zúñiga y León 2017) sobre competencias científicas e mediação pedagógica. Especificamente, este trabalho evocou o objetivo de caracterizar a mediação pedagógica que docentes utilizam na sua prática cotidiana, para o desenvolvimento de competências científicas em alunos do $9^{\circ}$ ano (do terceiro ciclo da educação geral básica). A pesquisa baseou-se no paradigma naturalista e uma metodologia que pressupõe um tipo de pesquisa mista, na qual os dados qualitativos e quantitativos são verificados a partir da observação e do questionário, usado para se obter uma análise mais profunda. A pesquisa foi realizada com a colaboração de 17 professores de ciências da Regional Heredia (divisão de zonas escolares, proposta pelo Ministério da Educação da Costa Rica), dispostos a colaborar, de modo que 


\begin{abstract}
os resultados obtidos se aplicaram apenas a esse grupo, sem poder generalizar. Dentre os principais resultados destaca-se que o papel, a metodologia (estratégias-recursos), o ato educativo e a avaliação utilizadas pelo corpo docente do terceiro ciclo tendem a estar mais próximos de um modelo tradicional de ensino dirigido a transmissão do conhecimento. Da mesma forma, é evidente que, como a mediação se concentra principalmente no modelo tradicional, os conhecimentos conceituais e científicos sobre procedimentos e atitudes são priorizados no planejamento, avaliação e mediação.
\end{abstract}

Palavras chaves: Mediação pedagógica; conhecimento científico; ciência; ensino fundamental; professores; competências científicas.

\title{
Introducción
}

La sociedad actual demanda del proceso educativo una constante renovación para responder a los diferentes avances de índole científico y pedagógico que acontecen diariamente, por lo cual el proceso educativo, en general, y en particular el de enseñanza de las ciencias debe desarrollar aprendizajes para la vida.

Hoy día, tal como lo menciona Marco-Stiefel (2002):

No sólo se hace preciso conocer los hechos o descodificar los mensajes, si no prestarles funcionalidad, con el fin de hacerlos puntos de partida para la integración de nuevos conocimientos. Los ciudadanos del mañana además de ver pasar la ciencia por sus vidas, han de ser capaces de mirar los acontecimientos científicos y tecnológico como algo que les afecta. (p. 33)

En este sentido, la educación debe abocarse al desarrollo de estas habilidades a fin de garantizar en el estudiantado un desarrollo personal y social óptimo. En este contexto se podrían rescatar dos ideas que plantea Bauman (2007) en las que sostiene que las consecuencias de estos desafíos implican nuevas formas de mirar la actuación humana. Dado que hemos sido formados con modelos y concepciones estáticas fundamentadas en el equilibrio (modernidad sólida), es necesario cambiar y migrar hacia modelos menos equilibrados e inestables.

Si bien se debe respetar la historia y los aportes que el modelo educativo tradicional ha hecho a la sociedad, en la actualidad sigue existiendo una insatisfacción por los resultados generados de este modelo. Pues se sigue mencionado que existe poca contextualización en los procesos formativos, así como una inadecuada formación de competencias científicas para la vida que representen verdaderas transformaciones individuales, colectivas y sociales (Tejada, 2008).

Además, tal como menciona Tejada (2007), existe poco aprovechamiento y mucho desconocimiento del desarrollo científico y tecnológico, así como de su utilidad en pro de dar soluciones reales para el bienestar individual, colectivo y social; situación que presume la 
doi: http://dx.doi.org/10.15359/ree.23-2.5

URL: http://www.una.ac.cr/educare

CORREO: educare@una.cr

necesidad de generar nuevas estrategias para la formación personal, ciudadana y profesional. De allí que, efectivamente, se puede mejorar y es legítimo en estas condiciones, confrontar la posibilidad de cambio.

Esto se puede resumir de la siguiente manera: está emergiendo un nuevo modelo de educación, que nos da pistas para redefinir lo que enseñamos en la escuela y que le da sentido a la formación del profesorado. Una ciencia que es una construcción humana realista, que es comprensiva, que está fundamentada en valores que cambian y que forman parte de los consensos de la comunidad científica (Quintanilla et al., 2013).

En ese contexto se pueden destacar dos elementos como valiosos en la enseñanza de las ciencias: el conocimiento científico y la mediación pedagógica desarrollada en el aula. En este caso, el proceso educativo debe desarrollar las herramientas conceptuales, procedimentales y actitudinales sobre las cuales se construye la educación científica, particularmente de la enseñanza de las ciencias, cuyo marco se encuentra en la formación científica de docentes, y en los planes y programas de estudio propuestos.

En este sentido, el personal docente ha de proponerse como mediador, capaz de generar una interacción pedagógica, social, dialógico, lúdico, consciente, intencional, sistemático, destinado a generar experiencias de aprendizaje integral, es decir, que permita desarrollar conocimientos y que posibilite el desarrollo de las potencialidades humanas en el ser, hacer, conocer y convivir (León, 2014).

Así, este nuevo modelo pedagógico debe buscar que el estudiantado sea responsable de su propio aprendizaje, desarrolle autonomía, asuma un rol activo en el aprendizaje, reflexione sobre lo que hace y utilice la tecnología como recurso útil para el aprendizaje (Jabif, 2007).

Para lograr lo antes expuesto, el colectivo docente ha de poseer un sólido conocimiento científico que lo faculte para crear y recrear ese proceso dinámico que es la enseñanza de las ciencias. Proceso que exige a la persona profesional de las ciencias una aproximación y cierta similitud con los grupos científicos que la construyen; a fin de crear modelos menos enciclopédicos, más intuitivos y funcionales, que permitan el acceso a la creciente terminología científica, a los conceptos y metodología propios de la ciencia y al desarrollo de competencias científicas para la vida.

De esta forma, la mediación pedagógica, así como los conocimientos científicos, son un elemento fundamental para lograr desarrollar competencias para el pensamiento científico, crítico y reflexivo en el estudiantado, adecuado a las necesidades científicas de la sociedad actual.

Al respecto, algunas investigaciones realizadas tanto a nivel nacional como internacional han mostrado la pertinencia y relevancia de estudiar en profundidad, cómo docentes y estudiantes, a la luz de sus modelos educativos, responden a estas nuevas exigencias formativas. 
En materia de competencias científicas existen estudios previos, como el de Zúñiga, Leiton y Naranjo (2011), quienes abordaron el desarrollo de las competencias científicas y sus diferentes dimensiones (cognitiva, procedimental y actitudinal) en un estudio comparativo entre Costa Rica y Argentina, en el que se señala que las competencia científicas de estudiantes, en su mayoría, se encuentran en un nivel bajo de desarrollo, así como que existe un marcado desinterés por la parte estudiantil por ampliar sus conocimientos científicos. En esa misma temática, Quintanilla (2012) hizo una recopilación de investigaciones, metodologías y concepciones que han de ser tomadas en cuenta para el desarrollo de competencias científicas, dentro de las que se señala la necesidad de redireccionar los procesos de formación científica, en virtud de buscar nuevas formas de mejorar los procesos de enseñanza aprendizaje de las ciencias, en aras de desarrollar competencias para el pensamiento científico útiles en la vida cotidiana de la ciudadanía.

En cuanto a las competencias científicas y la enseñanza de las ciencias a nivel nacional, se identificaron estudios como el realizado por Alvarado, Salas, Zúñiga, León y Torres (2015), quienes indagaron acerca de cómo los modelos de enseñanza de química, física y biología podrían generar, o no, competencias científicas para la vida en estudiantes de undécimo nivel. Así mismo se identificó el trabajo realizado por Padilla-Canales, Brooks-Calderón, Jiménez-Porras y Torres-Salas (2016), en el cual realizan un análisis de las competencias científicas propuestas en los planes de estudio de Biología, Física y Química de educación diversificada del Ministerio de Educación Pública, y su relación con el desarrollo científico tecnológico del país. A partir de estos se pudo concluir que existe cierto desconocimiento disciplinar, así como pedagógico por parte de docentes de los contenidos de las ciencias que se abordan en la educación secundaria. De igual forma, a partir de esta investigación, se establece que existe una disparidad entre las políticas país en términos de formación científica y el diseño e implementación de los currículos de ciencias naturales que dificultan el desarrollo de las competencias científicas en el estudiantado.

En relación con la mediación y la enseñanza de las ciencias, se resalta la investigación realizada por León (2015), sobre el malestar docente y la mediación pedagógica en la enseñanza de la física en Costa Rica. Donde se señala que la mediación pedagógica desarrollada por docentes de Física se puede calificar como aceptable, regularmente deficiente y deficiente, en la opinión estudiantil. Asimismo, el estudio evidenció que existe relación entre el nivel de malestar docente y la mediación pedagógica desarrollada por el personal docente en el aula.

En esta misma línea, el Informe del Estado de la Educación en Costa Rica 2010 ofrece una visión general del desarrollo y estado actual de la educación científica. En este se señala que existe una necesidad de fortalecer los programas de formación de las personas docentes de ciencias en todos los niveles del sistema educativo nacional, pues se debe entender que hay una gran diferencia entre saber ciencias y el tener capacitación para ser buenos o buenas docentes de ciencias. Así mismo se menciona la necesidad de ampliar las vivencias curriculares que favorezcan el entendimiento de las ciencias para lograr idear rutas posibles (estructuración de 
doi: http://dx.doi.org/10.15359/ree.23-2.5

URL: http://www.una.ac.cr/educare

CORREO: educare@una.cr

los programas de estudio, implementar programas de apoyo, acercamientos a la realidad, entre otros) para contribuir al desarrollo de la educación científica en el país y de ahí complementar el desarrollo pleno de las capacidades humanas y entornos amigables con el ambiente (Alvrado y Villegas, 2010).

De manera general, estas investigaciones muestran que existe una debilidad en los procesos de formación científica que se desarrollan en las aulas, por lo que establecer con claridad cuáles son esos factores asociados a estas debilidades se transforma en una tarea prioritaria de las casas de formación, a fin de poder generar estrategias que puedan mejorar las practicas pedagógicas del colectivo docente, así como fortalecer el conocimiento de los contenidos y habilidades científicas que desarrolla.

\section{Docentes y su mediación en la enseñanza de las ciencias}

En la actualidad, la ciencia se concibe como el medio de avance de la humanidad, por tal motivo es importante el papel que desempeñan sus docentes en la enseñanza de las ciencias naturales y el proceso de mediación pedagógica,

... ya que se convierten en el puente entre el conocimiento del docente y el conocimiento del estudiante, de ahí la importancia que el docente esté a la vanguardia en los aspectos pedagógicos y didácticos que surjan en cuanto a la enseñanza se refiere. (Espinosa, 2009, p. 21)

\section{Mediación pedagógica}

Es un proceso de gran relevancia en la educación, se basa en saber utilizar los contenidos y la forma de tratar diferentes temas con el fin de hacer del proceso de enseñanza y aprendizaje un momento de gran interés para el alumnado, en el que es un actor que puede opinar y expresar sus experiencias, enriqueciendo la clase, además donde pueda utilizar su creatividad para hacer más agradable este proceso.

Al respecto, Gutiérrez y Prieto (2004) conceptúan medicación pedagógica como "... el tratamiento de contenidos y de las formas de expresión de los diferentes temas a fin de hacer posible el acto educativo, dentro del horizonte de una educación concebida como participación, creatividad, expresividad y relacionalidad" (p. 50).

De igual manera Pérez (2009) menciona que la mediación pedagógica parte de la necesidad de promover experiencias de aprendizaje, de lograr la interactividad del estudiantado de manera integral como ser humano, comprender que el acto pedagógico no puede ser directivo, es una forma de interacción entre personas con niveles de experiencias diferentes, donde se parte de la necesidad de compartir para construir en equipo, en un determinado contexto. 
Propiciar en las aulas una mediación pedagógica en busca de la construcción de mejores conocimientos requiere romper con la idea de que educación es transmisión de conocimientos del personal docente hacia sus estudiantes, es necesario creer y practicar una educación donde el diálogo, la análisis crítico, el debate y las experiencias significativas sean prácticas fundamentales y diarias. De tal manera que el estudiantado se asuma como un actor y cada docente como guía del proceso de enseñanza aprendizaje.

Entonces, como lo propone León, 2014:

la mediación pedagógica está caracterizada por la relación dinámica del estudiante, con sus pares, el docente y el medio social que le rodea, donde el educador es quien orienta intencionalmente la actividad, para que el joven construya aprendizajes que le permitan auto organizar sus ideas con el fin de que le sean útiles en su cotidianeidad y al enfrentar nuevas experiencias. (p. 143)

\section{Rol docente}

En las últimas décadas se ha hecho aún más evidente la necesidad de asumir retos que coadyuven en la formación idónea de profesionales de las ciencias, dado que la sociedad requiere personas con un perfil innovador continuo, profesionales altamente competentes en términos educativos, socio-económicos y culturales, debe tener la capacidad para poner en práctica sus conocimientos, para utilizar alternativas metodológicas que faciliten el aprendizaje, pero que también permitan generar aprendizajes situados. Además, esta persona debe desarrollar formas de interacción, con respeto hacia otros seres humanos, su cultura y su espiritualidad. También debe desarrollar la capacidad para pensar en forma crítica y autónoma. Aún más, aprender a construir su propio proyecto de vida en forma armónica con la sociedad y el ambiente del cual es parte.

En ese particular, León (2014) propone algunas características que debe tener el cuerpo docente para mediar el proceso de aprendizaje:

- Persona capaz de crear espacios de interrelación, intercambios de conocimiento, diálogos donde el estudiantado sea partícipe en el acto educativo.

- Establece metas donde sus estudiantes sean partícipes.

- Favorece la perseverancia, hábitos de estudio, autoestima y la metacognición.

- Posee la intención de crear en el estudiante y la estudiante un aprendizaje significativo.

- Debe potenciar el desarrollo de saberes conceptuales, procedimentales y guía en procesos actitudinales.

- Emplea diferentes tratamientos pedagógicos según las demandas del colectivo estudiantil.

- Desarrolla en el estudiantado actitudes positivas: haciéndole vivir unos valores para que los haga operativos en su conducta dentro de su realidad sociocultural. 
doi: http://dx.doi.org/10.15359/ree.23-2.5

URL: http://www.una.ac.cr/educare

CORREO: educare@una.cr

\section{Conocimiento científico}

La sociedad actual demanda una educación científica más vivencial y contextualizada, pero también humanizada, es decir, que contribuya con el avance científico y tecnológico, sin dejar de lado la sostenibilidad de los recursos; en ese caso, debe contribuir a que los seres humanos desarrollen habilidades para convivir juntos, que les permitan aplicar saberes conceptuales y procedimentales, pero que también tengan un sentido de responsabilidad por el planeta (actitudinales).

Estos cambios requieren un conocimiento científico menos tradicional, no solo centrado en el saber por saber, ya que la humanidad demanda una visión más amplia, sistémica, crítica y reflexiva.

Desde esta visión, es importante que el conocimiento científico siga respondiendo a las necesidades del ser humano del siglo XXI; enmarcadas dentro de una sociedad globalizada e influenciada por las TIC, pero con rostro humano.

Esta nueva perspectiva concuerda con las ideas de Quintanilla (2005), quien propone:

Requiere enseñar a pensar los fenómenos del mundo con teoría, no podemos pensar en la actividad científica sólo como una actividad experimental en la que llevamos a los estudiantes a manipular instrumentos, tubos de ensayo, microscopios o lo que sea, sin entender qué modelo teórico se moviliza a través de esta actividad experimental. (p. 17)

Eldesarrollocientífico tecnológico demandan cambios en la formación científica, de modo que sea posible formar una ciudadanía capaz de ser competitiva en un mundo globalizado. Para ello, la población debe ser formada con un profundo conocimiento en los avances científicos y tecnológicos, pero a la vez con una visión humanista, con una sólida formación axiológica y espiritual de manera que se pueda garantizar mayores oportunidades de progreso, y bienestar personal y social.

\section{Base teórica conceptual de la competencia científica}

Las competencias científicas son el conjunto de experiencias tanto teóricas como prácticas que le permiten al ser humano responder ante las demandas de la vida (Zúñiga et al., 2011).

Puede ser distinto de una habilidad, a una motivación o a un prerrequisito en otro contexto. Esta competencia hace referencia a varias dimensiones:

- Dimensión saber, que permite comprender, identificar, conocer, ser capaces de caracterizar tipologías, de identificar teorías de forma diferente de la que se ha venido haciendo. 
- La dimensión saber hacer, comprende habilidades y competencias relacionadas con el campo de los procedimientos, por ejemplo, adaptar, imaginar, desarrollar procesos prácticos, diseñar actividades experimentales, saber aplicar; es decir, saber transferir a un contexto similar o distinto un conocimiento que es coherente con ese saber en ese otro contexto.

- La dimensión de ser se refiere al conjunto de actitudes que mueven a las competencias valóricas, sentido ético de la actividad científica, pensamiento holístico, que permiten al alumnado ser capaz de pensar de manera divergente.

Fundamentado en esta perspectiva teórica, el presente artículo expone los principales hallazgos de la investigación en cuanto a la mediación de las competencias científicas que realizan docentes de ciencia, este estudio se desarrolló en el marco del proyecto de investigación interdisciplinario entre la Escuelas de Ciencias Biológicas y División de Educología, de la Universidad Nacional, los resultados que se muestran se fundamentaron en uno de los objetivos propuestos: caracterizar la medición pedagógica y los conocimientos científicos que utilizan docentes en sus prácticas para el desarrollo de las competencias científicas en el III ciclo de educación General Básica. De manera que este estudio aporte un primer diagnóstico o sirva de fundamento a otras investigaciones que permitan implementar y coordinar acciones para el mejoramiento de la educación científica costarricense.

\section{Metodología}

La metodología seguida en este trabajo investigativo se inscribe en el enfoque mixto dominante propuesto por Hernández, Fernández y Baptista (2006), quienes mencionan que "en este modelo, el estudio se desarrolla bajo la perspectiva de alguno de los dos enfoques, el cual prevalece, y la investigación mantiene un componente del otro enfoque" (p. 773). En este caso el enfoque dominante fue el cualitativo pues se parte de la realidad educativa del colectivo docente de ciencias y de sus experiencias, complementado con la descripción y el uso herramienta SPSS.

Esta responde a un diseño fenomenológico que hace posible la interpretación de los procesos sociales, desde la propia perspectiva de la persona, es decir, examina la forma en la que se experimenta el mundo (Rojas, 2008); en ese caso, la mediación pedagógica, el desarrollo de las competencias científicas y los conocimientos científicos con los que cuenta el colectivo docente y que utiliza en el desarrollo de sus clases.

Para realizar la investigación se seleccionó el personal docente del III ciclo de Educción General Básica, específicamente se contó con el aporte de 17 docentes que al momento de realizar la recolección de la información se desempeñaban como profesionales de la educación 
doi: http://dx.doi.org/10.15359/ree.23-2.5

URL: http://www.una.ac.cr/educare

CORREO: educare@una.cr

en secundaria en la asignatura de ciencias, del circuito 01 y 02 de la Dirección Regional de Heredia, de ellos uno privado y el resto públicos, esta muestra fue seleccionada intencionalmente por acceso y disponibilidad de colaborar.

Para recopilar los datos se utilizó un cuestionario dirigido a docentes, el cual se aplicó presencialmente, contenía 47 preguntas cerradas. Este cuestionario estuvo conformado por cuatro dimensiones: a) Tratamiento del contenido para la que se incluyeron 32 ítems, que contemplaban aspectos relacionados con tipos de recursos didácticos y técnicas; modelos educativo; estrategias de mediación; planificación y evaluación, b) formas de expresión para la que se contemplaron 4 ítems en los que se incluyeron aspectos relacionados con las formas de comunicación y las relaciones de poder en el aula y c) acto educativo en el que se incluyeron 11 ítems con indicadores como: la participación del educando, la creatividad y el contexto, acto educativo, desarrollo de la clase.

Así mismo, para lograr profundizar en la dinámica de aula y las propuestas de mediación vivenciadas por los grupos docentes se realizó una observación no participante al desarrollo de 2 lecciones de cada docente, para lo cual se construyó una guía de observación en la que se contemplaron las mismas dimensiones e indicadores del cuestionario, y una dimensión más relacionada con las competencias científicas (conceptual, procedimental y actitudinal) constituida por 9 ítems, a fin de contrastar los datos.

Por otra parte, los instrumentos fueron validados por criterio experto, se consultó a 4 personas académicas con grado de doctorado y maestría, con vasta experiencia en investigación, en el campo de la didáctica de las ciencias. Entre los aspectos que se buscó validar se encontraban los siguientes: evaluar si realmente recogía la información de acuerdo con los objetivos de la investigación; examinar si las instrucciones, el vocabulario y los ítems eran comprensibles; detectar la existencia de preguntas o ítems que tenían poca utilidad para los objetivos de la investigación, así como evaluar si las categorías eran exhaustivas y mutuamente excluyentes. Una vez analizadas las sugerencias y recomendaciones se incluyeron en el instrumento las que se consideraron pertinentes.

De igual manera se le aplicó el coeficiente de confiabilidad Alfa de Cronbach, cuyos valores respectivamente fueron 0,8 para el cuestionario y 0,9 para la escala Linker de la observación, considerados como bueno y excelentes, según el criterio de fiabilidad.

El análisis de datos se realizó a partir de las dimensiones e indicadores de los instrumentos antes descritos, utilizando la herramienta SPSS para agilizar la interpretación, ya que si bien la muestra es poca, la estructura de los instrumentos fue amplia, por tal motivo los resultados de los datos se muestran agrupados a partir de los indicadores descritos, solo en algunos casos se presentan resultados específicos de los criterios. Este análisis estadístico se complementó con la descripción. De igual modo, se validó el análisis mediante la triangulación tanto de los instrumentos, equipo investigador, como a nivel teórico. 


\section{Análisis}

Dado que el eje de la investigación se centró en caracterizar la mediación pedagógica que utiliza el personal docente en su práctica cotidiana para el desarrollo de las competencias científicas en estudiantes de $9^{\circ}$ año y sus conocimientos científicos, en este apartado se desglosarán los resultados, acorde con las dimensiones e indicadores del estudio:

Dimensión tratamiento de contenidos: direccionada a identificar y caracterizar los siguientes indicadores: tipos de recursos didácticos y técnicas, modelos educativos, estrategias, planificación y evaluación.

Dimensión formas de expresión: orientada a caracterizar las formas de comunicación (lenguaje adecuado) que utilizan docentes en el desarrollo de sus clases.

Dimensión acto educativo: en la que se contemplaron como indicadores la participación del educando, creatividad, contexto y desarrollo de la clase.

Dimensión competencias científicas: orientada a evaluar las habilidades de docentes para el tratamiento de los contenidos conceptuales, procedimentales y actitudinales de las ciencias.

A continuación, se presentan los resultados y la discusión de la perspectiva docente y la observación en cuanto a la mediación pedagógica y el desarrollo de conocimiento científico.

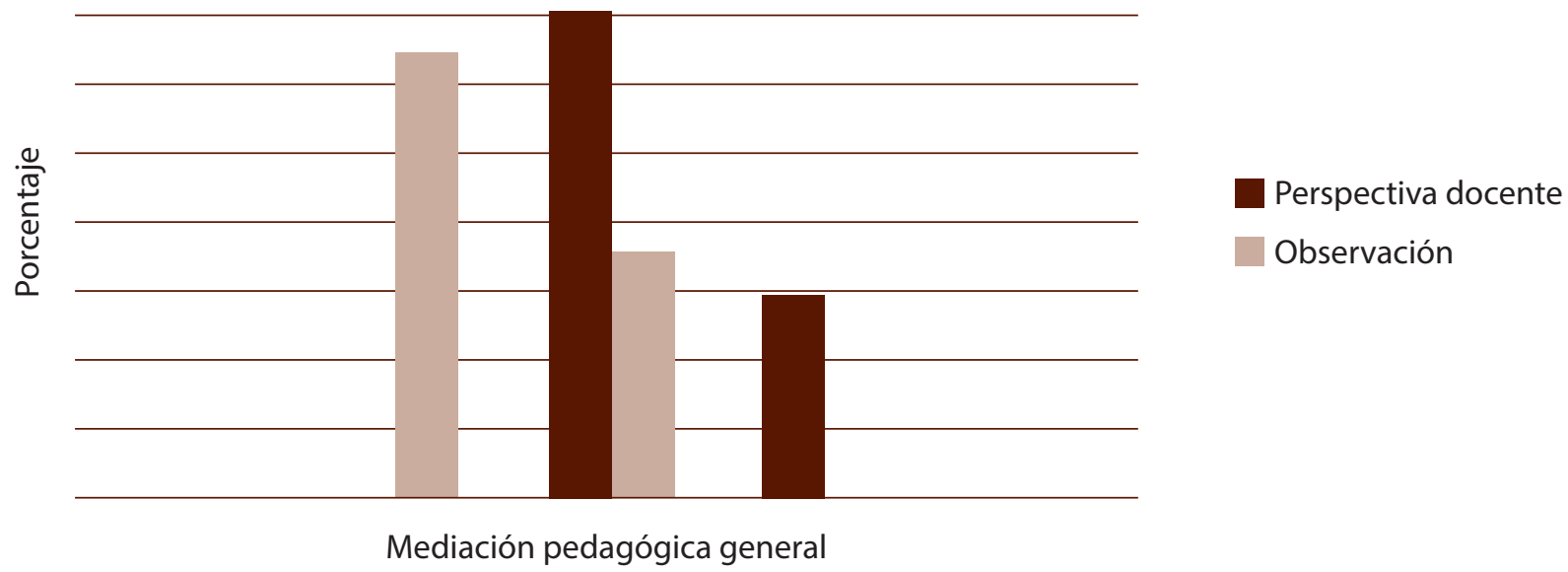

Figura 1: Total de mediación pedagógica agrupado, perspectiva docente (cuestionario) y observación de clase. Nota: Elaboración propia. 
doi: http://dx.doi.org/10.15359/ree.23-2.5

URL: http://www.una.ac.cr/educare

CORREO: educare@una.cr

Tal como se muestra en la Figura 1, la mediación pedagógica es valorada de manera diferente por el personal docente de noveno encuestado, por cuanto los grupos profesionales de la educación científica califican de manera mayoritaria (70 \%) su mediación como buena, empero las personas investigadoras la definen mayoritariamente (65\%) como deficiente. Estas valoraciones se presentaron en cada una de las dimensiones e indicadores de la mediación, las cuales se proponen a continuación.

Dimensión tratamiento del contenido, en cuanto a los recursos didácticos y las técnicas, se les consultó al grupo de profesionales de la educación a cerca de los recursos, técnicas y se observó cuáles de estas se utilizaban con mayor frecuencia.

El colectivo docente refirió utilizar variedad de recursos didácticos en sus clases tales como: pizarra, internet, laboratorios, libros, fotocopias, videos, esquemas demostraciones, entre otros, los cuales les ayudan a dinamizar y contextualizar los aprendizajes.

En contraste, al realizar las observaciones se encontró que los recursos mencionados por los docentes y las docentes no eran empleados con frecuencia y en los casos en lo que sí se apoyaban en ellos, no se les daba el manejo adecuado; por ejemplo, al usar la pizarra, el tamaño de la letra era muy pequeño, lo cual dificultaba la lectura; de igual manera se presentaron casos en los cuales la pizarra se encontraba escrita previo inicio de clase con una guía de preguntas que debía completarse a partir del libro de texto, de igual manera se identificó la subutilización de recursos, tal es el caso del proyector de multimedia, ya que 14 de las 17 instituciones visitadas poseían ese recurso; sin embargo, solamente 2 personas proyectaron presentaciones.

La utilización de variedad de recursos se circunscribe a un grupo menor de profesores y profesoras, tal fue el caso de una persona que le solicitó al colectivo estudiantil diseccionar flores de diferentes especies, utilizando equipo casero, realizar un video del proceso, elaborar un mapa conceptual y exponer a sus pares el trabajo realizado. Este tipo de metodología vivencial, evidencia que no se requiere equipo sumamente sofisticado para mediar las clases; de igual manera, se resalta la variedad de actividades respondiendo con ello a la diversidad de la clase, así como la integración del estudiantado al desarrollo de las lecciones de manera que sea responsable de su propio aprendizaje, desarrolle autonomía, asuma un rol activo en el aprendizaje, reflexione sobre lo que hace y utilice la tecnología como recurso útil para el aprendizaje (Jabif, 2007); de igual forma, que se promueva, trabajo cooperativo, reflexión, exploración, aplicación, procesos propios de la ciencia.

Según Torres, Mora, Garzón y Ceballos (2013), no utilizar variedad de recursos limita las habilidades exploratorias y creativas de estudiantes, lo que impide el desarrollo de competencias científicas, ya que la posibilidad de explorar al máximo otras formas de aproximación al conocimiento y construir en equipo aprendizajes se restringe de alguna manera, al utilizar un único recurso didáctico. 
En este sentido, se evidenció a partir del análisis comparativo y las observaciones realizadas que los docentes y las docentes centran sus procesos educativos en el uso de recursos como el libro de texto, sin complementarlos con imágenes o explicaciones en la pizarra, existe una contradicción por la parte docente de ciencias, por cuanto en el indicador tipos de recursos didácticos y técnicas dicen utilizar en un $94 \%$ diferentes recursos, imágenes y esquemas para dar las clases, demostraciones, laboratorios, sin embargo, en una pregunta específica sobre la utilización del libro de texto el $41 \%$ asume que usan ese recurso, en este caso esta postura fue afirmada en la observación donde en un 53 \% de las ocasiones se evidenció el uso el libro de texto, vale resaltar que no se condena el uso de ese recurso, lo que se debe mejorar es la forma en que se implementó, dado que el rol docente se resumió en indicar las páginas del libro de texto que se debían desarrollar. En la mayoría de los casos no se profundizó, no se revisaron ni explicaron las temáticas abordadas en el libro de texto. Por su parte, se observaron clases de una persona profesional de la educación que trabajó con el libro, empero, lo complementó con imágenes y explicaciones en la pizarra, de igual manera revisó el trabajo de sus estudiantes de manera general.

En este mismo sentido se les consultó y observó para identificar las estrategias que utilizaban para desarrollar sus clases; al respecto, es posible establecer que los grupos docentes reconocen desarrollar sus clases a partir de estrategias como clases magistrales, el trabajo en grupo y la resolución de problemas en las que tanto lo observado como lo manifestado por docentes parece coincidir. No obstante, estrategias como proyectos de investigación, exploraciones fuera del aula, trabajos de laboratorio, seminarios, debates, a pesar de que el personal docente manifestó que algunas veces las utilizaba, mientras que el dato que resulta de la observación muestra que son las estrategias menos utilizadas.

Se puede inferir que estos cuerpos docentes están desarrollando sus clases desde las perspectivas más tradicionales de la enseñanza, en las que las experiencias de aula se encuentran centradas en clases magistrales que utilizan como recurso el libro de texto o dictado, con ello se limita la apertura de espacios de aula en los que se promueva la reflexión desde la acción, el análisis, la comprensión de conceptos científicos, la metodología científica; todas estas habilidades que pueden ser promovidas a través de la utilización sistemática de estrategias y recursos como proyectos de investigación, trabajos exploratorios fuera del aula o experiencias de laboratorio en las que estudiantes hagan, piensen, escriban y encuentren sentido al hecho científico y desarrollen tanto destrezas manipulativas como intelectuales.

Por ejemplo, en una institución, la persona profesional de la educación aportó lecturas referentes a los tubérculos, tales como la papa, yuca, entre otros; sin embargo, después de realizar la lectura, no se contrastaron los aprendizajes, ni se llegó a una conclusión.

La estrategia más utilizada fue la del aprendizaje autónomo, donde el estudiantado resolvía prácticas individualmente en libros de texto, si bien, lo anterior permite abarcar contenidos, al ser una tarea estructurada de forma emisión-recepción, esto le confiere un papel pasivo al estudiantado, en menoscabo de aprendizajes para la vida. La ausencia de un tratamiento de 
doi: http://dx.doi.org/10.15359/ree.23-2.5

URL: http://www.una.ac.cr/educare

CORREO: educare@una.cr

contenido dinámico y contextualizado le asigna al alumnado un papel pasivo, que a la postre se puede ver reflejado en un bajo nivel de significancia de los conocimientos científicos.

En cuanto al uso de estrategias y recursos y al desarrollo de habilitadas científicas, existe una disparidad de criterios entre lo que se observa y lo que manifiesta el personal docente pues plantea que el tipo de recursos, así como las estrategias que utiliza son buenas para desarrollar habilidades relacionadas con el conocimiento científico; no obstante, las observaciones realizadas a sus clases indican todo lo contrario, situación que se respalda con lo antes discutido en este apartado y que hace suponer que se sigue propiciando el desarrollo de aprendizajes orientados a lo conceptual.

Según Quintanilla (2012), las estrategias para lograr generar una competencia científica son aquellas que permiten contextualizar el contenido visto en clase, como estudios de caso, resolución de problemas actuales, contextos históricos y prácticas de campo, las cuales permiten que el estudiantado, en el momento de afrontar problemáticas fuera del aula, logre resolverlas asertivamente utilizando el conocimiento científico. No obstante, como se mencionó anteriormente, según lo observado en el aula, las estrategias utilizadas no permiten la generación de conocimientos científicos, si bien, se puede lograr la capacidad cognitiva por medio de estrategias magistrales, el aprendizaje requiere un proceso vivencial y experimental, el cual no se observó durante la investigación.

Lo anterior va de la mano con el modelo educativo que impera en sus clases, por cuanto para el $53 \%$ prevalece el modelos emisión recepción, en complemento el $47 \%$ dice utilizar en sus clases modelos educativos más constructivos, tales como resolución de problemas, por descubrimiento entre otros. Por su parte, en las observaciones de clase, se determinó que el $71 \%$ implementa el modelo emisión recepción y en muy pocos ocasiones los otros modelos en promedio el $20 \%$ de los casos. La ausencia de un tratamiento de contenido dinámico y contextualizado, le asigna al estudiantado un papel pasivo, el cual a la postre se puede ver reflejado en un bajo nivel de significancia de los conocimientos científicos.

Otro de los indicadores analizados fue la evaluación, en este caso nuevamente se presenta disparidad en los criterios emitidos, una mayoría $65 \%$ de docentes dice partir de los conocimientos de sus estudiantes, realizar diferentes tipos de evaluaciones, retomar los visto en clase; sin embargo, en la observación se pudo contrastar cómo, cuando evalúan, lo hacen en su mayoría de manera sumativa, por lo que el proceso como tal se valoró como malo para el $76 \%$.

Las formas de evaluación que fomentan competencias científicas son aquellas que le permitan al estudiantado poner en práctica las capacidades y habilidades desarrolladas, por ejemplo, la resolución de problemas, dar ejemplos o aplicar en la vida cotidiana los saberes, investigación de problemas que involucren los conocimientos previos, o bien, aquellas que permitan evidenciar la formación de habilidades pertenecientes a actitudes, como clasificar residuos, reducir el uso de recursos, fomentar estilos de visa saludables, entre otros. Todos ellos 
evaluados tanto de manera sumativa como formativa a partir de debates, ensayos, proyectos o investigaciones, que además de contener el elemento conceptual y procedimental, involucran la postura o visión del estudiante sobre el tema, es decir, el procedimental (Cañal, 2012).

En relación con la planificación, nuevamente se contrastan las posturas, ya que profesionales de la enseñanza de las ciencias aducen realizar una adecuada planificación de sus clases $76 \%$, empero el $65 \%$ de las ocasiones se observó una improvisación, en algunas oportunidades se le consultaba al grupo por dónde iban en sus clases (trabajo del libro), esto se manifestaba en el poco aprovechamiento de la clase.

Tal como lo indican Prieto et al. (2006), para desarrollar competencias científicas se debe partir de una prefijación que le permita al estudiantado tener una participación activa del proceso educativo, guiada y coordinada sistemáticamente, es decir, donde se inicie con una situación problemática o interrogante que le estimula sobre qué debe aprender, cuándo hacerlo, dónde indagar, así como una forma de aplicación. Este eje permite aprender en profundidad y con autonomía, desarrollando competencias; sin embargo, durante la observación, esto se logró en muy pocas oportunidades.

En la Figura 2 se presentan los resultados de la dimensión formas de expresión y el indicador formas de comunicación (lenguaje adecuado).

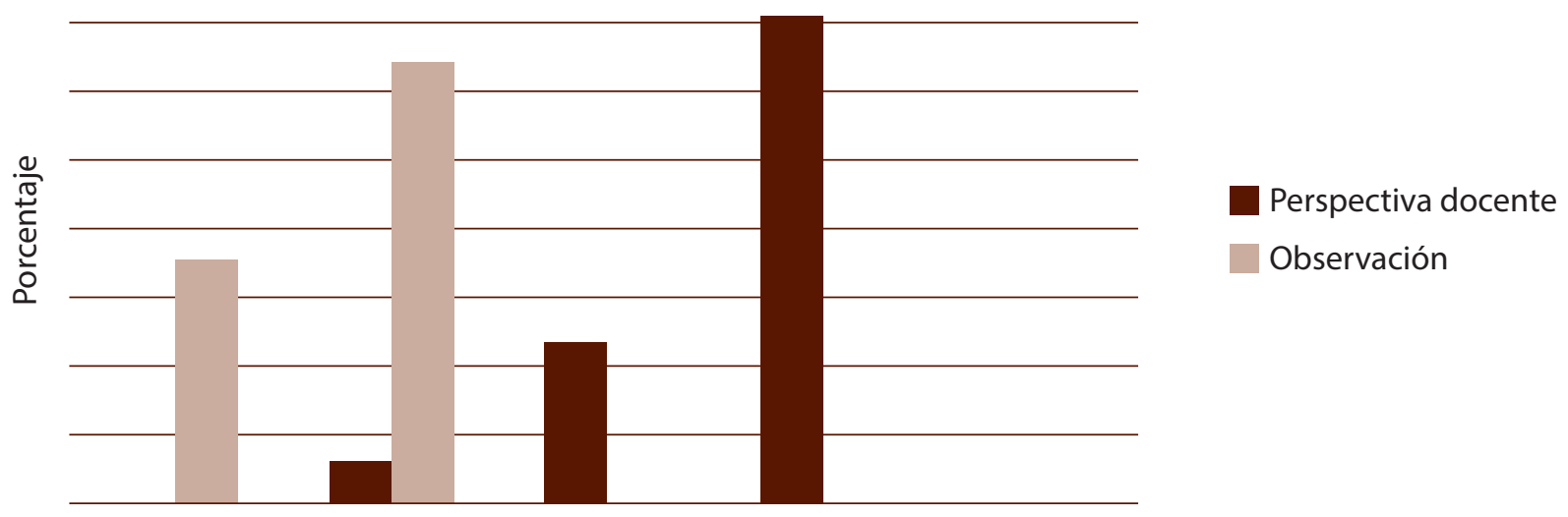

Formas de comunicación

Figura 2: Dimensión: Formas de expresión Indicador: Formas de comunicación (lenguaje adecuado) agrupado, perspectiva docente (cuestionario) y observación de clase.

Nota: Elaboración propia.

En este caso tenemos valoraciones porcentuales muy semejantes: $71 \%$ y $65 \%$, pero posturas diferentes, los docentes y las docentes aducen utilizar en clase un lenguaje científico claro y preciso, establecer espacios para que se dé una comunicación fluida estudiante- 
doi: http://dx.doi.org/10.15359/ree.23-2.5

URL: http://www.una.ac.cr/educare

CORREO: educare@una.cr

docente y docente-estudiante, y realizar intervenciones utilizando argumentaciones científicas; sin embargo, en el desarrollo de las lecciones se observó que el lenguaje utilizado era muy coloquial, en aquellos momentos donde se dio intervención docente, dado que una parte se queda en el escritorio realizando diferentes actividades (computadora), dejando de lado su rol mediador del conocimiento científico.

Se debe resaltar que el lenguaje es el medio principal con que cuenta la persona docente para articular los saberes, dado que permite potenciar saberes conceptuales y procedimentales mediante la trasmisión oral y escrita. Así mismo, otra de las competencias que se puede desarrollar, es la actitudinal, por cuanto estas se adquieren por el modelaje. La comunicación entre estudiante-estudiante y estudiante-docente beneficia procesos como la interacción, el debate, el intercambio de intereses, entre otros.

A la luz de los resultados identificados, se debe reflexionar sobre la importancia de potenciar espacios de comunicación formal e informal en el acto educativo, tanto para promover conocimientos científicos, como para generar relaciones humanas.

Por su parte, la dimensión: acto educativo indicador: participación educando, creativa y contexto, se muestra en las Figura 3, en este caso se mantienen valoraciones porcentuales semejantes $59 \%$ y $65 \%$, pero difieren las calificaciones, el grupo de docentes de la investigación refiere proponer actividades que permitan al estudiantado aplicar sus conocimientos en la resolución de problemas del contexto y realizarlo desde la interdisciplinaridad, actividades que permiten desarrollar el conocimiento científico, sin embargo, en la observaciones realizadas esos componentes no se presentaron en un $65 \%$ de los casos, por cuanto en el desarrollo de las lecciones no se daban ejemplos, a pesar de que en varios de las temáticas se podía salir al patio y desarrollar algunas actividades, hasta realizar laboratorios en la clase, dado que el espacio físico utilizado para trabajar eran laboratorios que cuentan con equipo. De igual manera se resalta que los temas desarrollados mayoritariamente eran partes de la planta, tejidos animales vegetales, estructura y función de la célula, sistemas del cuerpo humano y sus enfermedades, temas que permitían contextualizar lo visto en clase, promoviendo una actitud positiva en sus estudiantes hacia el conocimiento científico, de manera que se desarrollen capacidades y actitudes propias del saber ser, conocer y convivir tales como la motivación, curiosidad y que les permita ser personas críticas, reflexivas y creativas.

La ausencia de contextualización se puede relacionar con el uso del libro de texto como principal recurso, sin mediación. Tal como se indicó, su uso no es considerado como inadecuado, lo que se debe mejorar es el intercambio de ideas entre el estudiantado y la persona docente, por cuanto se observó que una parte del cuerpo docente proponía guías de preguntas, donde lo que debía hacer es estudiantado era transcribir al cuaderno lo expuesto en el libro de texto. Contrario a lo indicado, se presentaron clases en las cuales sí se contextualizó, se dieron ejemplos de la vida cotidiana y hasta se desarrollaron demostraciones utilizando plantas y videos alusivos al tema en estudio. 
A la luz de los resultados obtenidos queda en evidencia que la mayoría del profesorado que participó del estudio le da más importancia a los contenidos que al desarrollo de habilidades y actitudes científicas; domina las metodologías tradicionales basadas en la transcripción de libros de texto a partir de una guía de preguntas, lo cual responde al modelo de enseñanza tradicional, donde el rol estudiantil es pasivo y solo debe responder a las interrogantes planteadas por el educador o educadora. No obstante, si se hace uso de estrategias más vivenciales como laboratorios, trabajo de campo, resolución casos entre otros, el estudiantado desarrollaría un aprendizaje metacognitivo, relacionando la nueva información con algún aspecto ya existente en la estructura cognitiva.

Costa Rica enfrenta desafíos especiales en este nuevo milenio y entre ellos, el referente al mejoramiento cualitativo de la educación científica. Como bien lo plantean los Informes del Estado de la Nación, el recurso humano laboral solo se ha preparado apropiadamente para competir en un mundo globalizado (saberes técnicos). Esto evidencia la necesidad de proponer alternativas y oportunidades para el estudiantado, que le permitan ser responsable de su propio aprendizaje, sea autónomo, asuma un rol activo en el aprendizaje, reflexione sobre él y su realidad inmediata. En general, que sea un ente transformador, eso implica un cambio sustancial en el rol docente identificado en esta investigación.

Para finalizar con las dimensiones relacionadas con la mediación pedagógica, a continuación se presentan los resultados obtenidos en cuanto al acto educativo indicador: desarrollo de la clase, las cuales se presentan a nivel gráfico en las Figura 3.

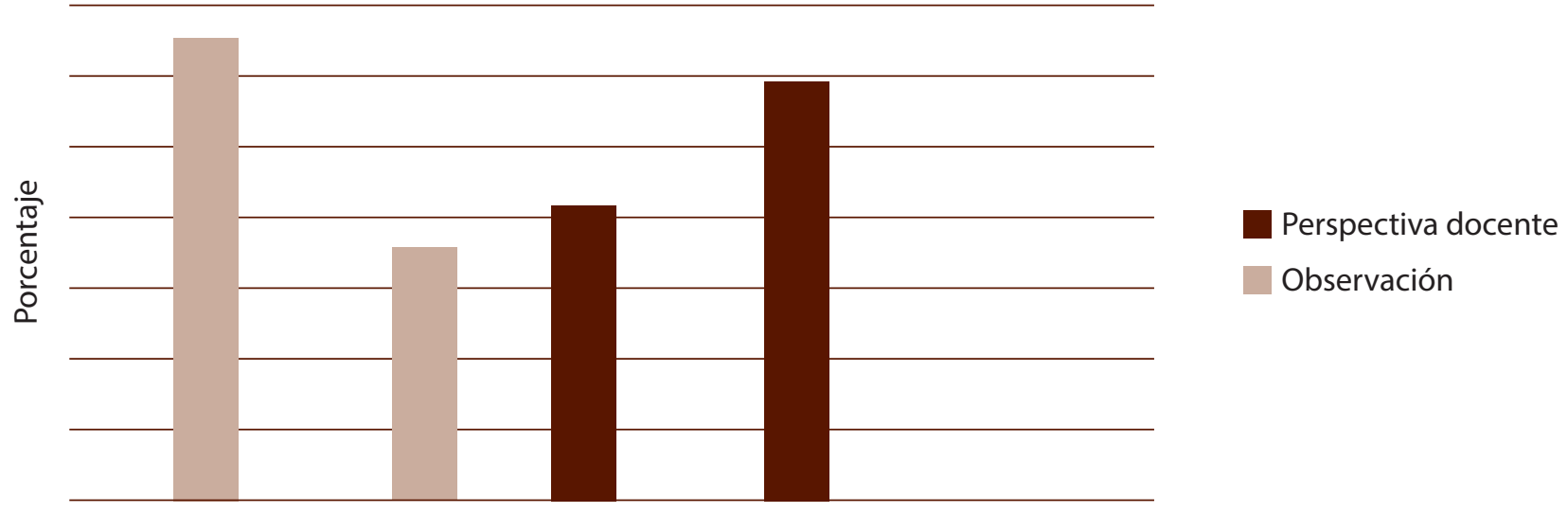

Acto educativo desarrollo de la clase

Figura 3: Dimensión: acto educativo. Indicador: desarrollo de la clase agrupado, perspectiva docente y observación. Nota: Elaboración propia. 
doi: http://dx.doi.org/10.15359/ree.23-2.5

URL: http://www.una.ac.cr/educare

CORREO: educare@una.cr

En relación con el rol docente, se puede observar en la Figura 3, como se mantienen los puntos de vista antes descritos, el grupo de docentes indica trabajar en el aula favoreciendo el intercambio de ideas y el debate entre grupos, expresando opiniones y sentimientos y verificando los objetivos propuestos; empero, cuando el personal docente sede el desarrollo de los aprendizajes a un recurso, sin explicación ni contextualización, lo antes expuesto es difícil de lograr, dado que las observaciones evidencian en un $77 \%$ de los casos que el personal docente no cumple con su rol de mediador del conocimiento científico.

De manera general se puede indicar que este abordaje pedagógico estático apoya únicamente la transmisión y la recepción de conceptos, no permite un aprendizaje integral y permanente en el individuo; de tal manera que no se da un desarrollo del pensamiento reflexivo y crítico de la realidad inmediata como lo propone el Ministerio de Educación Pública (MEP, 2017).

En consecuencia, tal como se indicó en la introducción, la actualidad demanda del proceso de enseñanza-aprendizaje de las ciencias, una mediación científica que busque la implementación de propuestas didácticas dinámicas y contextualizadas, pero que también responda a las exigencias del contexto y necesidades del alumnado como sujetos sociales, históricos y culturales (Pozo, 1997).

Continuando con las dimensiones e indicadores de la investigación, a continuación se presenta, en las Figuras 4 y 5, la dimensión competencia científica y el indicador conceptual, procedimental y actitudinal.

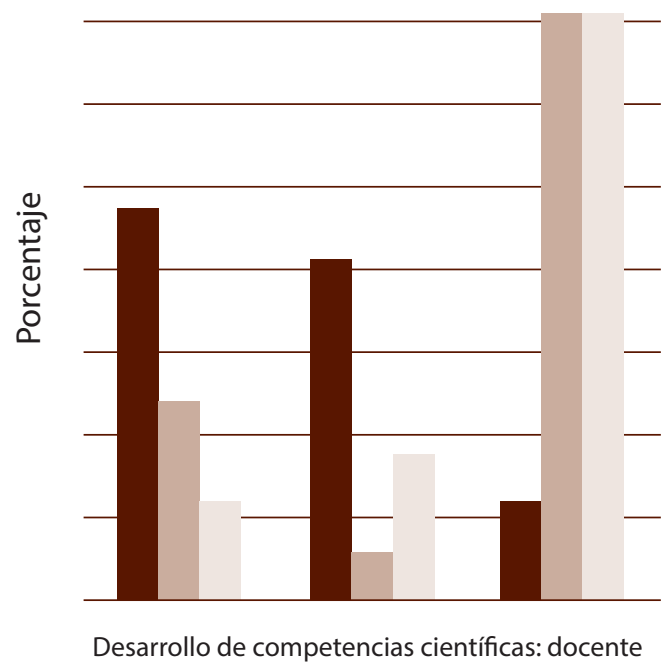

Competencia científica: conceptual

Competencia científica: procedimental

Competencia científica: actitudinal

Figura 4: Mediación de las competencias científicas en clase (agrupado): conceptual, procedimental y actitudinal postura del profesorado.

Nota: Elaboración propia. 
doi: http://dx.doi.org/10.15359/ree.23-2.5

URL: http://www.una.ac.cr/educare

CORREO: educare@una.cr

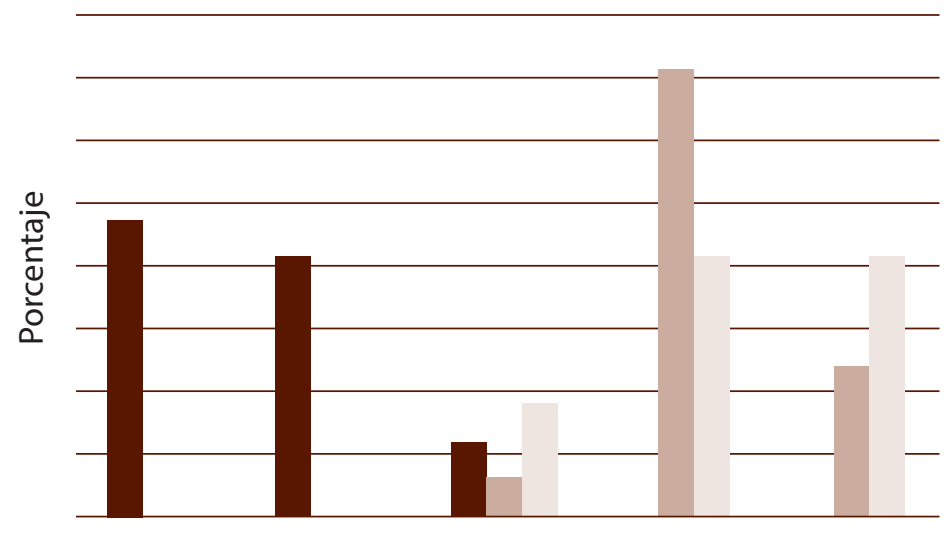

Desarrollo de la competencia científica: observación
Competencia científica: conceptual

Competencia científica: procedimental Competencia científica: actitudinal

Figura 5: Mediación de las competencias científicas en clase (agrupado): conceptual, procedimental y actitudinal observación.

Nota: Elaboración propia.

Deacuerdo con lo mostrado en las figuras anteriores se puede deducir que se mantienen los planteamientos descritos antes, el grupo docente dice desarrollar en su mediación pedagógica las tres dimensiones científicas (conceptual, procedimental y actitudinal); sin embargo, se contrasta con lo observado, donde la principal dimensión desarrollada es la conceptual, en la cual, como se evidencia en las Figuras 4 y 5, las posturas coinciden tanto por parte de docentes, como en lo observado en clase.

En cuanto a la competencia procedimental y actitudinal, existe una diferencia de criterio, por cuanto docentes refieren en un $71 \%$ potenciarla algunas veces; sin embargo, en la observación no se vio el desarrollo de esas dimensiones. Por su parte, en la observación se identificó cómo el $71 \%$ nunca desarrolla competencias científicas procedimentales, es decir, no utiliza instrumentos de medición y técnicas que permitan organizar y analizar la información, no evidencia en sus clases habilidades para la investigación ni la comunicación; lo anterior se contrasta con las preguntas relacionadas con el uso de recursos que potencien esa habilidad tales como: demostraciones, laboratorios actividades prácticas, dado que en promedio en un $94 \%$ de los casos no se reflejaron en las clases.

Las valoraciones antes descritas también obedecen a modelos tradicionales para la enseñanza de las ciencias, promoviendo con esto el desarrollo y concreción de aprendizajes más de corte conceptual que procedimental, por lo que se considera que este tipo de modelo de enseñanza no contribuye, en gran proporción, a que estudiantes sean capaces de desarrollar o reforzar competencias científicas de manera integral, ya que el saber hacer requiere, por lo general, poner en práctica una secuencia de pasos que se complementan con imágenes, videos, simulaciones, juegos o secuencia de acciones, entre otros, lo cual, en su mayoría, 
doi: http://dx.doi.org/10.15359/ree.23-2.5

URL: http://www.una.ac.cr/educare

CORREO: educare@una.cr

no se implementa en las clases, a pesar de que a nivel curricular los programas de estudio sugieren el tratamiento de habilidades que les permitan pensar, explorar, formular, manipular y experimentar en un espacio específico (MEP, 2017).

Acorde con lo observado, el estudiantado tiende a repetir los contenidos, sin comprender ni establecer vínculos entre los conocimientos científicos y las necesidades del mundo cotidiano. Lo anterior conlleva a una estimulación del aprendizaje memorístico y mecánico que obstaculiza el desarrollo de los conocimientos procedimentales relacionados con la investigación científica.

De forma similar, se presentaron los datos relacionados con la mediación de la dimensión actitudinal. En un $41 \%$ de los casos no se observó su promoción, a pesar de que las personas docentes refirieron algunas veces potenciarlas. Esas competencias no se logran por cuanto los cuerpos docentes no contrastan lo visto en clase con la realidad inmediata, o simplemente no le dan importancia a componentes actitudinales tales como el cuidado al medio ambiente ni a la salud, tal como se refirió en el apartado anterior, pese a que los temas desarrollados permitían potenciar actitudes, dejando inconclusa esa formación integral del estudiantado, ya que las actitudes permiten aplicar los conocimientos conceptuales y procedimentales a nivel personal y social.

En relación con lo anterior, es válido señalar que las estrategias y recursos utilizados no permitían desarrollar capacidades como la curiosidad, plantearse preguntas, observar, criticar, reflexionar y solucionar problemas. A esto se suma la ausencia en la planificación de espacios que permitieran la investigación, el poco o nulo uso de laboratorios y la poca interacción y exploración del medio natural, lo cual reduce de manera considerable el desarrollo de actitudes científicas.

La sociedad en la actualidad demanda de manera general una formación integral y de manera particular una ciencia fundamentada en valores (Quintanilla et al., 2013), ya que se requiere un ser humano con capacidad transformadora desde su contexto en la dialéctica individuo-sociedad para mejorar la calidad de vida. Por ello, la educación científica debe promover procesos de enseñanza y aprendizaje significativos y críticos, situación que se puede lograr promoviendo una mediación pedagógica integral, incorporando las diferentes habilidades (conceptuales, procedimentales y actutudinales).

No basta con aprender a repetir en un examen contenidos (conocer) y en algunos casos aprender a hacer, es fundamental también aprender a ser y aprender a vivir en relación con los demás, con el fin de lograr la autorrealización como ser humano y como parte de una sociedad que cada día demanda la solidaridad, el trabajo en equipo, de manera que se tenga una sociedad fundamentada en los valores y que, por tanto, nos permita ser mejores personas.

Tal y como se ha planteado con anterioridad, una de las principales características de las competencias se refiere a la capacidad de una persona para llevar a cabo una acción o enfrentar una determinada situación. En el caso de las competencias en la enseñanza de las ciencias, estas cualidades apuntan hacia el buen desempeño en el ámbito del conocimiento científico. No 
obstante, muchas veces estas competencias se ligan con la capacidad de que los estudiantes y las estudiantes sepan repetir, de memoria, actividades propias del modelo emisión recepción, que no responde a las necesidades actuales de mediación pedagógica, en los que se requiere personas capaces de trabajar en equipo, dialogar, consensuar; activas; reflexivas; críticas; analíticas, entre otras. Habilidades y competencias que se pueden potenciar y desarrollar si el personal docente es un mediador del conocimiento científico, si utiliza estrategias y recursos constructivos, acordes con las necesidades estudiantiles, si promueve una atmósfera de comunicación científica y asertiva, si implementa estrategias de evaluación integrales, acorde con las capacidades y ritmo de aprendizaje de su alumnado (León, 2014).

En suma, la mediación de las competencias científicas acorde con la observación se puede calificar como deficiente para un $65 \%$, apegada al modelo tradicional evitando que el estudiantado desarrolle un pensamiento científico desde las diferentes competencias, por cuanto solo se potencia el saber conceptual.

Los resultados anteriores coinciden con los hallazgos determinados por investigaciones como las de Alvarado et al. (2015) y Zúñiga et al. (2011), los cuales, de igual manera, resaltan la necesidad de una adecuada formación científica, donde se potencien las diferentes habilidades científicas.

\section{Algunas ideas para concluir}

De la interpretación de la información aportada por las personas colaboradoras, se tienen conclusiones y hallazgos relevantes para esta investigación, entre las que destacan:

Se evidenció que el profesorado centra su proceso educativo en un recurso como es el libro de texto, sin complementarlo con otros que permiten desarrollar conocimiento científico.

Las estrategias utilizadas por el personal docente fomenta principalmente el aprendizaje autónomo, lo que impide una dinámica de clase que favorezca el desarrollo de conocimientos científicos.

Se identificó que el cuerpo docente de ciencias del estudio no planifica ni evalúa teniendo en cuenta las competencias conceptuales, procedimentales y actitudinales.

Las personas profesionales de la investigación están desarrollando sus clases desde las perspectivas más tradicionales de la enseñanza, limitando con esto la apertura de espacios de aula en los que se promueva la reflexión desde la acción, el análisis y la comprensión de conocimientos científicos.

La mediación desarrollada en clase promueve las competencias conceptuales en su mayoría dejando de lado las procedimentales y actitudinales. Por cuanto se implementan y priorizan recursos, actividades y estrategias evaluativas tendientes al conocimiento, memorización de datos y hechos. 
doi: http://dx.doi.org/10.15359/ree.23-2.5

URL: http://www.una.ac.cr/educare

CORREO: educare@una.cr

La mediación pedagógica acorde con la investigación es calificada como deficiente, dado que no se da un proceso de aprendizaje vivencial, contextualizado ni creativo, el estudiantado se asume como un receptor de conocimientos teóricos. Lo anterior concuerda con las estrategias, recursos didácticos y evaluación que se implementan en clase, relacionados con el aprendizaje tradicional de las ciencias.

Cabe señalar que el proceso de investigación fue una oportunidad para diagnosticar la mediación pedagógica del conocimiento científico. De acuerdo con los resultados, se logró comprender que el abordaje educativo de la enseñanza de las ciencias en noveno, para la muestra en estudio, requiere ser mejorada. En ese sentido se recomienda al ente rector de la mayoría de esos centros educativos, propiciar espacios de capacitación y actualización, tanto del componente pedagógico como del científico.

\section{Referencias}

Alfaro, G. y Villegas, L. R. (2010). Tercer informe del estado de la educación. La educación científica en Costa Rica. San José, Costa Rica: CONARE.

Alvarado, A., Salas, R., Zúñiga, A., León, G. y Torres, M. I. (2015). Las competencias científicas y los modelos de enseñanza en el proceso de aprendizaje de biología, física y química: El caso de dos grupos de la secundaria costarricense. México: Red Durango de Investigadores Educativos. Recuperado de http://redie.mx/librosyrevistas/libros/competenciascientificas.pdf

Bauman, Z. (2007). Los retos de la educación en la modernidad liquidan. Madrid: Gedisa Editorial.

Cañal, P. (2012). ¿Cómo evaluar la competencia científica en secundaria? Alambique, 72, 7583. Recuperado de https://www.cad.unam.mx/programas/actuales/cursos diplo/ diplomados/uaem 2014/00 cont/09 material/material/02 modulo2/04 qui/material modulo II Quimica/04 Como Evaluar CompetenciaC.pdf

Espinosa, E. A. (2009). Los mediadores pedagógicos en la enseñanza de las ciencias: La implementación de un programa educativo multimedia en la enseñanza del sistema circulatorio. El Hombre y la Máquina. 32, 20-37. Recuperado de http://www.redalyc.org/ pdf/478/47811604003.pdf

Gutiérrez, F. y Prieto, D. (2004). La mediación pedagógic. Apuntes par una educación a distancia alternativa (10 ed.). Santiago, Guatemala: Ediciones La Copia Fiel.

Hernández, R., Fernández, C. y Baptista, M. P. (2006). Metodología de la investigación (4a ed.). México D.F.: McGraw-Hill. 
Jabif, L. (2007). La docencia universitarian bajo un enfoque de competencias. Orientaciones prácticas para docentes. Valdivia, Chile: Universidad Austral de Chile

León, G. (2014). Aproximaciones a la mediación pedagógica. Revista Calidad en la educación Superior, 5(1), 136-155. Recuperado de http://investiga.uned.ac.cr/revistas/index.php/ revistacalidad/article/view/348/249

León, G. (2015). Malestar docente y mediación pedagógica. Mexico: Instituto Universitario Anglo Español. Recuperado de http://iunaes.mx/wp-content/uploads/2015/06/LibroGiselle-2015-1.pdf

Marco-Stiefel, B. (2002). Alfabetización científica y enseñanza de las ciencias: Estado de la cuestion. En P. Membiela (Ed.), Enseñanza de las cencias desde la perspectiva cienciatecnologia-sociedad (pp. 33-46). Madrid: Narcea.

Ministerio de Educación Pública. (2017). Programas de estudio de ciencias de tercer ciclo de educación general básica. San José, Costa Rica: Autor. Recuperado de http://www.mep. go.cr/sites/default/files/programadeestudio/programas/ciencias3ciclo.pdf

Padilla-Canales, C., Brooks-Calderón, P., Jiménez-Porras, L. D. y Torres-Salas, M. I. (2016). Dimensiones de las competencias científicas esbozadas en los programas de estudio de Biología, Física y Química de la Educación Diversificada y su relación con las necesidades de desarrollo científico-tecnológicas de Costa Rica. Revista Electrónica Educare, 20(1), 1-26. doi: http://dx.doi.org/10.15359/ree.20-1.2

Pérez, R. A. (2009). El constructivismo en los espacios educativos. San José, Costa Rica: Coordinación Educativa y Cultural Centroamericana, CECC/SICA. Recuperado de https://drive.google. com/file/d/1F4JCV0KMPmQG4EJZuqayvYqSarTqF1LE/view

Pozo, J. (1997). Enfoques para la enseñanza de la ciencia (Capítulo VIII). En Pozo, J. (Autor), Teorías cognitivas del aprendizaje (pp. 265-308). Recuperado de http://www.geocities.ws/ javi her/lec 9b.pdf

Prieto, A., Barbarroja, J., Reyes, E., Monserrat, J., Díaz, D., Villaroel, M. y Álvarez-Mon, M. (2006). Un nuevo modelo de aprendizaje basado en problemas, el ABP 4x4, es eficaz para desarrollar competencias profesionales valiosas en asignaturas con más de 100 alumnos. Aula Abierta, 87, 171-194. Recuperado de http://didac.unizar.es/jlbernal/ensenar en la Universidad/ pdf/13 ABP.pdf

Quintanilla, M. (2005). Qué y cuáles son las competencias científicas. Identificación y caracterización de competencias científicas en el aula, ¿qué cambia en la enseñanza y en los nuevos modelos de conocimiento? En Foro Educativo Nacional. Competencias Científicas (pp. 13-30). Colombia: Ministerio de Educación Nacional. Resumen recuperado de http:// colombiaaprende.edu.co/html/mediateca/1607/articles-128237 archivo.pdf 
doi: http://dx.doi.org/10.15359/ree.23-2.5

URL: http://www.una.ac.cr/educare

CORREO: educare@una.cr

Quintanilla, M. (2012). La investigación en evaluación de competencias de pensamiento científico desde la formación continua del profesorado. Algunas directrices epistemológicas. En M. Quintanilla (Comp.), Las competencias de pensamiento científico desde las 'voces' del aula (pp. 15-46). Recuperado de https://www.researchgate.net/profile/Mario Roberto Gatica/ publication/280886383 Las competencias de pensamiento cientifico desde las voces del aula/links/55ca991308aeca747d69ece4.pdf

Quintanilla, M., Martínez, M., Manrique, F. y Reinoso, J. (2013). Identificación, caracterización y evaluación de competencias de pensamiento científico en profesores de ciencia en formación a través del enfrentamiento a la solución de problemas. En IX Congreso Internacional sobre investigación en didáctica de las ciencias (pp. 2901-2906), Girona. Recuperado de https://core.ac.uk/download/pdf/38988686.pdf

Rojas, L. P. (2008). Elementos conceptuales y metodológicos de la investigación cualitativa. San José, Costa Rica: Editorial UCR.

Tejada, A. (2007.) Desarrollo y formación de competencias: Un acercamiento desde la complejidad. Acción Pedagógica, 16(1), 40-47. Recuperado de https://dialnet.unirioja.es/ servlet/articulo?codigo $=2968571$

Tejada, A. (2008). Analise de un modelo integral baseado no paradigma da complexidade para a compreesao, definicao, avaliacao e aplicacao das competencias (Tesis de doctorado). Instituto de Psicologia, Universidade de São Paulo, São Paulo.

Torres, Á., Mora, E., Garzón, F., y Ceballos, N. E. (2013). Desarrollo de competencias científicas a través de la aplicación de estrategias didácticas alternativas. Un enfoque a través de la enseñanza de las ciencias naturales. Tendencias, 14(1), 187-215. Recuperado de https:// dialnet.unirioja.es/servlet/articulo?codigo $=4453237$

Zúñiga, A. Leiton, R. y Naranjo, J. A. (2011). Nivel de desarrollo de las competencias científicas en estudiantes de secundaria de (Mendoza) Argentina y (San José) Costa Rica. Revista Iberoamérica de la Educación, 56(2), 1-12. Recuperado de http://rieoei.org/ deloslectores/4246Zuniga.pdf

Zúñiga, A, y León, G. (2017). Las competencias científicas en estudiantes del tercer ciclo (9) de educación general básica en la Región Central de Heredia circuito 01 y 02 de la Universidad Nacional (Informe 048514). Heredia: Vicerrectoría Académica, Universidad Nacional. Manuscrito inédito. 